\title{
FACTORES DE RIESGO SOCIODEMOGRÁFICO DE IDEACIÓN E INTENTO SUICIDA EN ADULTO MAYOR. BOGOTÁ, COLOMBIA 2012-2013
}

Recibido: junio del 2018

Aceptado: marzo del 2019

Natalia Carantón Macías ${ }^{1}$, Andrea Sarmiento Traslaviña ${ }^{1}$, Gustavo Jaimes Monroy², Giovane Mendieta Izquierdo ${ }^{3}$

\section{Resumen}

Objetivo. Describir los factores de riesgo sociodemográfico de ideación e intento suicida en el adulto mayor en Bogotá, años 2012-2013. Método. Estudio observacional descriptivo transversal de datos comunes de la base oficial del sistema de vigilancia epidemiológica de la conducta suicida (Sisvecos) de la Secretaria Distrital de Salud de Bogotá, en 2012-2013. Se utilizó el cálculo porcentual, OR y Chi-cuadrado de Pearson para procesar los datos con un nivel de significancia de $\mathrm{p}<0,05$. Resultados. La mayoría de los casos se presentan entre 60 a 64 años (34,52\%); sexo femenino (69,44\%); aseguramiento en salud (49,60\%) que pertenecían al régimen subsidiado, se afectan más los casados con $(6,75 \%)$ para ideación y $(4,76 \%)$ para intento suicida. Se evidenció la relación entre las variables: sexo (Chi-cuadrado $=6,87 ; p=0,009$ ); estrato bajo (Chi-cuadrado $=9,733$; $\mathrm{p}=0,002)$ y ocupación (Chi-cuadrado $=4,047 ; \mathrm{p}=0,044)$. Se encontró un riesgo 4,4 veces mayor de presentar intento e ideación suicida en personas que viven en estratos bajos que aquellos que viven en estratos altos (OR $=4,359$, IC de $95 \%$ y $1,618-11,745)$; se encontraron OR protectores en ocupación por fuera de la casa $(\mathrm{OR}=0,393$, IC de $95 \%$ y 0,156 - 0,991) y ser de sexo masculino (OR de 0,469, IC de $95 \%$ y 0,246 - 0,831). Conclusiones. Se encontró significancia estadística para ideación e intento suicida en el adulto mayor en relación con estudio y sexo $\mathrm{P}=0,009$; estrato bajo $\mathrm{P}=0,002$ y ocupación $\mathrm{P}=0,044$. Existe 4,35 veces mayor riesgo de intento e ideación en estratos socioeconómicos bajos respecto a los altos. Las actividades fuera del hogar y vivir en pareja se identifican como factor protector.

Palabras clave: adulto mayor, ideación suicida, intento de suicidio, suicidio

\footnotetext{
${ }^{1}$ Maestría en Salud Pública y Desarrollo Social de la Fundación Universitaria del Área Andina.

${ }^{2}$ Docente investigador de la Facultad de Odontología de la Universidad Antonio Nariño.

3 Profesor asistente de la Facultad Educación y Humanidades de la Universidad Militar Nueva Granada.
} 


\section{FACTORS OF SOCIODEMOGRAPHIC RISK OF SUICIDAL THINKING AND ATTEMPT IN ELDERS. BOGOTA, COLOMBIA 2012-2013}

Natalia Carantón Macías ${ }^{1}$, Andrea Sarmiento Traslaviña', Gustavo Jaimes Monroy², Giovane Mendieta Izquierdo ${ }^{3}$

\section{Abstract}

Objective: To describe the sociodemographic risk factors of suicidal thinking and acting in elders in Bogotá, between 2012 and 2013. Method: Cross-sectional observational and descriptive study about common data in the official database of the epidemiologic assessment system of suicidal behavior (sisvecos in Spanish) from Bogota's Health Bureau, in 2012-2013. We used percentual estimations and Pearson's chi-squared test to process data with a significance level of $\mathrm{p}<0,05$. Results: Most cases occur in people of ages between 60 to 64 years old (34,52\%); females $(69,44 \%)$; health insurance subsidized by the government $(69,44 \%)$. From the ones who were married, there were those with suicidal thoughts $(6,75 \%)$ and those who had attempted suicide (4,76\%). Relationship between factors was the following: sex (chi-squared $=6,87 ; \mathrm{p}=0,009$ ); low social class (chi-squared $=9,733 ; \mathrm{p}=0.002$ ) and occupation (Chi-squa$\mathrm{red}=4,047 ; \mathrm{p}=0,044)$. We found a risk of suicidal thought and attempt 4.4 times higher in people who live in low-income neighborhoods than those who live in higher-income neighborhoods (OR $=4,359,95 \% \mathrm{CI}$ and 1,618 - 11,745). Finally, protective OR's were found to be either occupation outside home $(\mathrm{OR}=0-393,95 \%$ and $0,156-0,991 \mathrm{CI})$ and being of male sex ( $\mathrm{OR}=0,469,95 \%$ and $0,246-0,831 \mathrm{CI})$. Conclusions: $\mathrm{A}$ statistical significance was found for suicidal thoughts and attempts in the elder in relation with education and sex $\mathrm{P}=0,009$; low income bajo $\mathrm{P}=0,002$ and occupation $\mathrm{P}=0,044$. There is a risk 4.35 times higher of attempt and thought in low-income neighborhoods against high-income neighborhoods. Any activities outside home and living with a partner were identified as protective factors. 


\section{FATORES DE RISCO SOCIODEMOGRÁFICO DE IDEAÇÃO E TENTATIVA DE SUICÍDIO EM IDOSO. BOGOTÁ, COLÔMBIA 2012-2013}

Natalia Carantón Macías ${ }^{1}$, Andrea Sarmiento Traslaviña ${ }^{1}$, Gustavo Jaimes Monroy², Giovane Mendieta Izquierdo ${ }^{3}$

\section{Resumo}

Objetivo: descrever os fatores de risco sociodemográfico de ideação e tentativa de suicídio em idoso em Bogotá, em 2012 e 2013. Método: estudo observacional descritivo transversal de dados comuns da base oficial do sistema de vigilância epidemiológica do comportamento suicida (Sisvecos) da Secretaria Estadual de Saúde de Bogotá, em 2012 e 2013. Foi utilizado o cálculo porcentual, OR e Qui-Quadrado de Pearson para processar os dados com um nível de significância de $\mathrm{p}<0,05$. Resultados: a maioria dos casos se apresenta entre 60 e 64 anos (34,52\%); sexo feminino (69,44\%); asseguramento em saúde (49,60\%), que pertenciam ao regime subsidiado; são mais afetados os casados com $6,75 \%$ para ideação e 4,76\% para tentativa de suicídio. Tornou-se evidente a relação entre as variáveis: sexo (Qui-Quadrado $=6,87 ; \mathrm{p}=0,009$ ); classe social baixa ( $Q$ ui-Quadrado $=9,733 ; \mathrm{p}=0,002$ ) e ocupação (Quo-Quadrado = 4,047; $\mathrm{p}=0,044)$. Foi verificado um risco 4,4 vezes maior de apresentar ideação e tentativa de suicídio em pessoas que moram em lugares de classe baixa do que os que vivem em lugares de classe alta $(\mathrm{OR}=4,359$, IC de $95 \% \mathrm{e}$ 1.618 - 11.745); foram constatados OR protetores em ocupação fora de casa $(\mathrm{OR}=0,393$, IC de $95 \%$ e 0,156 - 0,991) e ser de sexo masculino (OR de 0,469 , IC de $95 \%$ e 0,246 - 0,831). Foi verificada significância estatística para ideação e tentativa de suicídio em idoso em relação ao estudo e ao sexo $(P=0,009$; classe baixa $P=0,002$ e ocupação $P=0,044)$. Existem 4,35 vezes mais riscos de ideação e tentativa de suicídio em classes socioeconômicas baixas em comparação com as altas. As atividades fora de casa e morar com companheiro(a) são identificadas como fator protetor. 


\section{Introducción}

El fenómeno del envejecimiento en la población es una realidad de la cual Colombia no es ajena (1). La Organización de las Naciones Unidas (ONU) estima que el número de personas mayores de 60 años en el mundo pasará de seiscientos millones en el 2000 a mil doscientos millones en el 2025 y a casi dos mil millones en el 2050 (2).

El envejecimiento se estudia como un proceso continuo y dinámico que inicia con el nacimiento y se desarrolla a lo largo de la vida, es la etapa después de los 60 años de edad que termina con la muerte; también se le conoce con los términos vejez, senectud, ancianidad o tercera edad (3). El comportamiento demográfico en la actualidad en Europa y América ha cambiado, la base de la pirámide se ha ido estrechando con ampliación simultánea en la punta; la tendencia demográfica lleva a la disminución de la población infantil e incremento de los adultos mayores, especialmente el aumento de los más viejos; situación conocida como transición demográfica $(4,5)$. En Colombia, al año 2050 se espera que el total de la población se acerque a 72 millones, con una esperanza de vida ligeramente superior a los 79 años y con más del $20 \%$ por encima de 60, lo cual se traducirá en una estructura de la pirámide poblacional en forma rectangular (5).

Debido al impacto que tiene en las comunidades y países enteros, el suicido es considerado un problema de salud

66 pública $(6,7)$, una tragedia personal que termina con la vida de un indivi- duo y que tiene un efecto de onda continua que perturba drásticamente la vida de las familias, amigos y comunidades (6). En el marco de las lesiones autoinflingidas se documenta que la tasa de suicidio en países desarrollados es mayor en la población adulto mayor (8). Desde la perspectiva de género, el suicidio es más frecuente en hombres mayores de 50 años, respecto a las mujeres y después de los 85 años es diez veces mayor (8).

Para el año 2014, la OMS estimó 804.000 muertes en todo el mundo, lo que representa una tasa anual estandarizada por edad de 11,4 suicidios por cada 100.000 habitantes (15,0 para los hombres y 8,0 para las mujeres). Las personas mayores de 70 años hacen parte de las altas tasas de suicidio en casi todas las regiones del mundo (6). En las dos últimas décadas del siglo XX, las tasas de suicidio en Colombia fluctuaron entre tres y cinco por cada cien mil habitantes con tendencia a la alza, sobresale el grupo de edades entre $15 \mathrm{y}$ 24 años en mujeres y por encima de 60 años en los hombres (9).

En el periodo 1973-1996, la mayor proporción de muertes causadas por suicidio se presentó en Colombia, en el grupo de 15-24 años para las mujeres con 3,25 por cada 100.000 habitantes; en el caso de los hombres las tasas más altas se presentaron en mayores de 60 años, con 12,6 casos por 100.000 habitantes (10), aspecto reportado en estudios posteriores $(3,6-9,11)$ situación que evidencia que la relación hombre-mujer respecto al suicidio va en aumento (11), el mayor riesgo de suicidio en Colombia ocurre 
en hombres en edad productiva, adultos mayores y en regiones poco desarrolladas, el riesgo de mortalidad por suicidio ha aumentado cuatro veces más en hombres, adultos jóvenes y adultos mayores (12).

En Bogotá, entre 2010 y 2014 , se presentaron 108 casos de suicidio consumado en la población adulta mayor, donde 18 casos fueron mujeres y 90 hombres (13). El suicido consumado es característico en los hombres de edad avanzada con métodos más agresivos como ahorcamiento o herramientas corto punzantes, a diferencia de los intentos suicidas en las mujeres, donde se involucran métodos como la intoxicación exógena (14). El intento suicida implica actos que provocan daño físico, que no son letales y que pueden o no requerir atención médica $(15,16)$; es decir, que se sobrevive a cualquier acto de autoagresión con poco $\mathrm{o}$ alto potencial letal.

Por otra parte, dentro de las principales causas reportadas en la literatura de ideación suicida en el adulto mayor se encuentran: factores sociales (17) como el aislamiento y exclusión social (18); enfermedad psiquiátrica (17); conflicto intergeneracional en la co-residencia que puede llegar a generar estrés y llevar a conductas suicidas (19) y discapacidad funcional asociada a los deseos de muerte (20).

Los pacientes de edad avanzada con enfermedades físicas tienen altos índices de ideación suicida y de comportamiento de autodestrucción $(21,22)$, condiciones que llevan a depresión acompañada de ideación suicida, as- pectos que adicionalmente generan una carga social y económica significativa para las comunidades, lo que aumenta la atención de los servicios de salud (23), razón por la que se recomienda ampliar las formas de diagnóstico y tratamiento asertivo de la depresión en los ancianos (24).

Como factores protectores se asocian las situaciones personales que generan razones para vivir, los programas de prevención del suicidio para los ancianos (25) y la atención primaria basada en comunidad (26). Las personas que intentan suicidarse están en mayor riesgo de morir, frente a los que no han presentado intentos de suicidio $(6,14)$; por cada suicidio consumado hay más personas que lo intentan, siendo este el factor de riesgo más importante; estudios señalan que por cada adulto que murió por suicidio puede haber al menos otros 20 intentos $(6,14)$. El objetivo del artículo es describir los factores de riesgo sociodemográfico de ideación e intento suicida en el adulto mayor en Bogotá, entre 2012 y 2013.

\section{Método}

Se realizó un estudio observacional descriptivo transversal que permitió identificar los factores de riesgo sociodemográfico de ideación e intento suicida en el adulto mayor en Bogotá, 2012-2013. Para este estudio se emplearon registros de información secundaria y se utilizó la base oficial del sistema de vigilancia epidemiológica de la conducta suicida (Sisvecos) de la Secretaria Distrital de Salud de Bogotá, de la cual se extrajeron las variables dependientes: ideación e 
intento de suicidio, y las variables sociodemográficas independientes, que para nuestro estudio se determinaron: grupo etario adulto igual o mayor de 60 años, sexo, estado civil, estrato social, área de ocurrencia del caso o empresa social del Estado (ESE) notificadora, régimen de afiliación y ocupación. El total de casos para intento e ideación señalados en la ciudad fue de 9.244 casos con todas las formas relacionadas con suicidio para todas las edades. El análisis se realizó con la totalidad de los casos registrados en mayores de 60 años como intento e ideación suicida para los dos años (2012 y 2013) y este correspondió a 252 casos ( $\mathrm{n}=179$ para ideación $\mathrm{y} \mathrm{n}=73$ para intento).

El análisis estadístico fue realizado con el software SPSS $20.0^{\circ}$ para Windows. Las variables cualitativas fueron expresadas como frecuencias absolutas, porcentajes e intervalos de confianza IC, y las cuantitativas con la media y la desviación estándar e IC. Se realizó análisis correlacional así: para comparar las variables cualitativas se utilizaron tablas de contingencia para análisis de Chi-cuadrado; el nivel de significancia se estableció como $\mathrm{p}<0,05$. Para la estimación del riesgo fue utilizado el Odds Ratio (OR), con las variables que podrían ser indicadoras de riesgo. Para su interpretación, frente a si es o no factor de riesgo, se consideró que OR mayor que la unidad es un factor de riesgo y si es menor que la unidad es de protección.

Para el análisis de Chi-cuadrado y el OR se recodificó la variable de: estrato socioeconómico como: estratos bajos para los estratos 1,2 y 3 , en donde 1 es el menor y estratos altos son 4,5 y 6 , donde 6 es el más alto. La variable de ocupación se recodificó para la permanencia de oficios o actividades dentro o fuera de la casa como: permanece en el hogar y no permanece en el hogar. Dado que existe una discusión legal en Colombia por la edad en la que se considera al adulto mayor en el grupo de la tercera edad (27), el recorrido de la muestra se utilizó para seleccionar la edad de análisis y se recodificó como: iguales o menores de 63 años e iguales o mayores de 64 años; el estado civil fue recodificado como: vive en pareja y no vive en pareja.

\section{Consideraciones éticas}

Se contempla la confidencialidad de la información y el anonimato como criterio fundamental de la ética y el principio bioético de la investigación. No se identifican posibles consecuencias o repercusiones negativas resultantes de la publicación del estudio, se protegió el anonimato y no se llevó a cabo ninguna intervención directa con la población objeto de investigación en concordancia con el Código de Helsinki (28) y los lineamientos de la Resolución 008430 de 1993 (29) del Ministerio de Salud en Colombia.

\section{Resultados}

Se analizaron 252 casos que correspondieron a: $\mathrm{n}=179$ para ideación $\mathrm{y} \mathrm{n}=73$ para intento, todos fueron atendidos $y$ confirmados por la Secretaría Distrital de Salud de Bogotá a través de sus diferentes hospitales (ESE). El grupo etario 
con mayor número de casos fue el de 60-64 años con $34,52 \%$ (87 casos), seguido de los adultos mayores de 65 a 69 con $25 \%$ (63 casos). El sexo que mayor número de casos presenta es el femenino con un total de $69,44 \%$ equivalente a 175 casos y en hombres $30,56 \%$ con 77 casos (tabla 1).

Tabla 1. Distribución por grupo etario de los adultos mayores que presentaron ideación o intento suicida

\begin{tabular}{c|c|c|c|c|cc}
\hline \multirow{2}{*}{ Edad (años) } & \multicolumn{2}{|c|}{ Mujeres } & \multicolumn{2}{c}{ Hombres } & \multicolumn{2}{c}{ Total } \\
\cline { 2 - 8 } & $\mathbf{N}^{\circ}$ & $\%$ & \multicolumn{1}{c}{$\mathbf{N}^{\circ}$} & $\%$ & $\mathbf{N}^{\circ}$ & $\%$ \\
\hline $60-64$ & 61 & 24,21 & 26 & 10,32 & 87 & 34,52 \\
$65-69$ & 49 & 19,44 & 14 & 5,56 & 63 & 25,00 \\
$70-74$ & 27 & 10,71 & 16 & 6,35 & 43 & 17,06 \\
$75-79$ & 21 & 8,33 & 10 & 3,97 & 31 & 12,30 \\
$80-84$ & 9 & 3,57 & 6 & 2,38 & 15 & 5,95 \\
85 o más & 8 & 3,17 & 4 & 1,59 & 12 & 4,76 \\
SD & 0 & 0,00 & 1 & 0,40 & 1 & 0,40 \\
Total & $\mathbf{1 7 5}$ & $\mathbf{6 9 , 4 4}$ & $\mathbf{7 7}$ & $\mathbf{3 0 , 5 6}$ & $\mathbf{2 5 2}$ & $\mathbf{1 0 0}$ \\
\hline
\end{tabular}

Fuente: elaboración propia.

Se realizó un análisis de Chi-cuadrado de Pearson para establecer la asociación entre las variables. Los resultados mostraron asociación entre sexo con intento e ideación suicida (Chi-cuadrado $=$
6,87; $p=0,009)$; entre el estrato, la ideación y el intento suicida (Chi-cuadrado =9,733; $\mathrm{p}=0,002)$; ocupación con intento e ideación suicida (Chi-cuadrado $=4,047 ; \mathrm{p}=0,044)($ tabla 2$)$. 
INVESTIGACIONES ANDINA No. 39, Vol. 21

Tabla 2. Chi-cuadrado calculado para intento e ideación suicida en adulto mayor

\begin{tabular}{|c|c|c|c|c|c|}
\hline Variable & Categorización & \multicolumn{2}{|c|}{$\begin{array}{c}\text { Intento e ideación } \\
\text { Número y } \\
\text { porcentaje }\end{array}$} & $\mathrm{Xi2}$ & $P$ \\
\hline \multirow{3}{*}{ Sexo } & Masculino & $46(25,70 \%)$ & $31(42,5 \%)$ & \multirow[t]{3}{*}{6,87} & \multirow[t]{3}{*}{0,009} \\
\hline & Femenino & $133(74,3 \%)$ & $42(57,5 \%)$ & & \\
\hline & Total & 179 (100\%) & $73(100 \%)$ & & \\
\hline \multirow{3}{*}{ Edad } & $\begin{array}{l}\text { Iguales o menores a } 63 \\
\text { años }\end{array}$ & $55(30,7 \%)$ & $21(26,8 \%)$ & \multirow[t]{3}{*}{0,094} & \multirow[t]{3}{*}{0,759} \\
\hline & $\begin{array}{l}\text { Iguales o mayores a } 64 \\
\text { años }\end{array}$ & $124(69,3 \%)$ & $52(71,2 \%)$ & & \\
\hline & Total & $179(100 \%)$ & $73(100 \%)$ & & \\
\hline \multirow{4}{*}{$\begin{array}{l}\text { Estrato } \\
\text { social }\end{array}$} & Estratos bajos 1,2,3 & $172(96,1 \%)$ & $62(84,9 \%)$ & \multirow[t]{3}{*}{9,733} & \multirow[t]{3}{*}{0,002} \\
\hline & Estratos altos 4,5,6 & $7(3,91 \%)$ & $11(15,1 \%)$ & & \\
\hline & Total & $179(100 \%)$ & $73(100 \%)$ & & \\
\hline & $\begin{array}{l}\text { No permanece en el } \\
\text { hogar }\end{array}$ & $12(18,2 \%)$ & $13(36,1 \%)$ & \multirow[t]{3}{*}{4,047} & \multirow[t]{3}{*}{0,044} \\
\hline \multirow[t]{2}{*}{ Ocupación } & Permanece en el hogar & $54(81,8 \%)$ & $23(63,9 \%)$ & & \\
\hline & Total & $179(100 \%)$ & $73(100 \%)$ & & \\
\hline \multirow{3}{*}{ Estado civil } & No vive en pareja & $105(58,7 \%)$ & $51(69,9 \%)$ & \multirow[t]{3}{*}{2,76} & \multirow[t]{3}{*}{0,97} \\
\hline & Vive en pareja & $74(41,3 \%)$ & $22(30,1 \%)$ & & \\
\hline & Total & 179 (100\%) & $73(100 \%)$ & & \\
\hline
\end{tabular}

Fuente: elaboración propia.

Para el riesgo (OR) los resultados arrojaron un riesgo 4,4 veces mayor de presentar intento e ideación suicida en personas que viven en estratos de bajos niveles socioeconómicos 1,2 y 3 , respecto a estratos altos 4,5 y $6(\mathrm{OR}=$ 4,359, IC de $95 \%, 1,618$ - 11,745). Los datos también indican que existe un ligero efecto protector cuando el adulto mayor tiene actividades fuera del hogar

70 respecto a permanecer en el hogar (OR =0,393, IC de $95 \%, 0,156-0,991)$. Así mismo, para este grupo etario se identificó la ideación y el intento suicida como factor protector; desde una perspectiva de género, del sexo masculino (OR = 0,469, IC de $95 \%, 0,246$ - 0,831). Estos datos se muestran contrarios a lo que reporta la literatura y podrían indicar que los hombres actúan de manera contundente al consumar el intento de suicidio. Las variables de vivir en pareja y de edad no presentaron resultados significativos (tabla 3 ). 
Tabla 3. OR calculado sociodemográfico para intento e ideación suicida en adulto mayor

\begin{tabular}{l|c|cc}
\hline \multirow{2}{*}{ Estrato social } & \multicolumn{3}{|c}{ Ideación } \\
\cline { 2 - 3 } & Valor del OR & Intervalo de confianza del 95\% \\
\hline $\begin{array}{l}\text { Estratos bajos / } \\
\text { Estratos altos }\end{array}$ & 4,359 & 1,618 & 11,745 \\
& & & 1,997 \\
$\begin{array}{l}\text { Menores de 63 años / } \\
\text { Mayores de 64 }\end{array}$ & 1,098 & 0,604 & \\
$\begin{array}{l}\text { No permanecer en el } \\
\text { hogar / } \\
\begin{array}{l}\text { Permanecer en el } \\
\text { hogar }\end{array}\end{array}$ & 0,393 & & 0,991 \\
$\begin{array}{l}\text { Vive en pareja / } \\
\text { No vive en pareja }\end{array}$ & 0,658 & 0,156 & 1,145 \\
& & & \\
$\begin{array}{l}\text { Masculino / } \\
\text { Femenino }\end{array}$ & 0,469 & & 0,831 \\
\hline
\end{tabular}

Fuente: elaboración propia.

Sobre los adultos mayores que presentaron ideación o intento de suicidio durante los años 2012 y $2013(\mathrm{~N}=252)$, su ocupación principal fue el hogar, lo que representa el 57,77\% $(\mathrm{N}=145)$; distribuido así: empleado $12,7 \%(\mathrm{~N}=32)$; desempleado o desocupado $8,3 \%(\mathrm{~N}=$ 21); jubilado o pensionado $6,7 \%(\mathrm{~N}=$ $17)$; independiente $4,3 \%(\mathrm{~N}=11)$; estudiante $4 \%(\mathrm{~N}=10)$; no aplica $4 \%(\mathrm{~N}=$ $10)$; sin dato $0,4 \%(\mathrm{~N}=1)$; otros trabajadores de servicios personales a particulares, no clasificados bajo otros epígrafes $2 \%(\mathrm{~N}=5)$ de la población.
Respecto al aseguramiento en salud, la población adulta mayor que presentó intento o ideación suicida en una proporción mayor pertenecen al régimen subsidiado 49,60\% ( $\mathrm{N}=125)$, seguido del contributivo $42,06 \%(\mathrm{~N}=106) \mathrm{y}$ $4,37 \%$ clasificados como vinculados. ( $\mathrm{N}$ =11). La ESE de la cuidad de Bogotá que más reportó eventos de ideación e intento suicida en el periodo analizado fue el Hospital Fontibón con un 14,29\% de los eventos; seguida del Hospital Rafael Uribe Uribe con un 13,10 \% y el Hospital Chapinero con $10,32 \%$ (tabla 4). 
INVESTIGACIONES ANDINA No. 39, Vol. 21

Tabla 4. Empresas Sociales del Estado notificadoras de los eventos de ideación o intento suicida en adultos mayores

\begin{tabular}{|c|c|c|c|}
\hline $\begin{array}{l}\text { ESE responsable de la } \\
\text { información }\end{array}$ & Frecuencia & Porcentaje & $\begin{array}{l}\text { Porcentaje } \\
\text { válido }\end{array}$ \\
\hline Hospital Fontibón & 36 & 14,29 & 14,29 \\
\hline Hospital Rafael Uribe Uribe & 33 & 13,10 & 13,10 \\
\hline Hospital Chapinero & 26 & 10,32 & 10,32 \\
\hline Hospital del Sur & 22 & 8,73 & 8,73 \\
\hline \multicolumn{4}{|l|}{ Hospital Vista Hermosa I } \\
\hline & & 7,54 & 7,54 \\
\hline Nivel Ese & 19 & & \\
\hline Hospital de Usaquén & 18 & 7,14 & 7,14 \\
\hline Hospital Pablo VI Bosa & 17 & 6,75 & 6,75 \\
\hline Hospital de Suba & 16 & 6,35 & 6,35 \\
\hline Hospital Engativá & 14 & 5,56 & 5,56 \\
\hline Hospital Centro Oriente & 13 & 5,16 & 5,16 \\
\hline Hospital San Cristóbal & 12 & 4,76 & 4,76 \\
\hline Hospital de Usme & & 4,37 & 4,37 \\
\hline Hospital Tunjuelito II Nivel & 11 & & \\
\hline Ese & 10 & & \\
\hline Hospital Nazaret & 5 & 1,98 & 1,98 \\
\hline Total & 252 & $100^{*}$ & ${ }^{*} 100$ \\
\hline
\end{tabular}

Fuente: elaboración propia.

En cuanto al estado civil, en los hombres casados se presenta el mayor porcentaje

72 tanto para ideación como para intento con $6,75 \%(\mathrm{~N}=17)$ y $4,76 \%(\mathrm{~N}=12)$ res- pectivamente. En las mujeres se presenta más ideación suicida en las viudas con el $17,86 \%(\mathrm{~N}=45)$, los intentos en las casadas con $4,76 \%(\mathrm{~N}=12)($ tabla 5$)$. 
Tabla 5. Relación de ideación, intento suicida, estado civil y sexo en adulto mayor

\begin{tabular}{|c|c|c|c|c|c|c|c|c|c|c|}
\hline \multicolumn{11}{|c|}{ Sexo } \\
\hline \multirow{3}{*}{ Estado civil } & \multicolumn{4}{|c|}{ Hombre } & \multicolumn{4}{|c|}{ Mujer } & & \\
\hline & \multicolumn{2}{|c|}{ Ideación } & \multicolumn{2}{|c|}{ Intento } & \multicolumn{2}{|c|}{ Ideación } & \multicolumn{2}{|c|}{ Intento } & \multicolumn{2}{|c|}{ Total } \\
\hline & $\mathbf{N}^{\circ}$ & $\%$ & $\mathbf{N}^{\circ}$ & $\%$ & $\mathbf{N}^{\circ}$ & $\%$ & $\mathbf{N}^{\circ}$ & $\%$ & $\mathbf{N}^{\circ}$ & $\%$ \\
\hline Soltero & 7 & 2,78 & 8 & 3,17 & 20 & 7,94 & 9 & 3,57 & 44 & 17,46 \\
\hline Casado & 17 & 6,75 & 12 & 4,76 & 26 & 10,32 & 12 & 4,76 & 67 & 26,59 \\
\hline Unión libre & 6 & 2,38 & 4 & 1,59 & 15 & 5,95 & 5 & 1,98 & 30 & 11,90 \\
\hline Separado & 9 & 3,57 & 2 & 0,79 & 25 & 9,92 & 7 & 2,78 & 43 & 17,06 \\
\hline Divorciado & 0 & 0,00 & 1 & 0,40 & 2 & 0,79 & 0 & 0,00 & 3 & 1,19 \\
\hline Viudo & 7 & 2,78 & 4 & 1,59 & 45 & 17,86 & 9 & 3,57 & 65 & 25,79 \\
\hline Total & 46 & 18,25 & 31 & 12,30 & 133 & 52,78 & 42 & 16,67 & 252 & *100 \\
\hline \multicolumn{5}{|c|}{ Ideación $P=0,102$} & \multicolumn{6}{|c|}{ Intento $P=0,506$} \\
\hline
\end{tabular}

Fuente: elaboración propia.

\section{Discusión}

En nuestro estudio se encontró que en Bogotá los adultos mayores que presentan ideación o intento suicida pertenecen en mayor proporción al grupo etario de 60 a 64 años con un 34,52\% de los casos (87 casos); seguido de los adultos mayores de 65 a 69 con $25 \%$ (63 casos), edades identificadas como de riesgo para estas condiciones $(10,14,30$, 31 ), los suicidios consumados son más comunes en los adultos mayores de 70 años $(7,12,30,32)$, donde los hombres mayores de 70 años poseen la tasa más alta de muertes por suicidio (12), lo que indica que en esta etapa de la vida son más efectivos los intentos $(6,30)$. Estos datos aportan a la explicación que la tendencia a disminuir la ideación y el intento de suicidio debería verse reflejado en un posible aumento de las cifras de sui- cidio consumado en mayores de 70 años $(6,12,14,32)$.

Encontramos que el análisis de las variables sexo y estrato socioeconómico en relación a intento e ideación evidencia que existe un riesgo 4,35 veces mayor de intento e ideación en estratos socioeconómicos bajos, aspecto descrito en estudios previos $(6,33)$, así como factor protector de las actividades fuera del hogar y vivir en pareja, situaciones descritas como razones para vivir (26). El mayor número de casos se presenta en estrato socioeconómico 2, con 20 casos en hombres $(7,94 \%)$ y 56 en mujeres $(22,22 \%)$, para un total de 76 reportes en este nivel socioeconómico. Seguido del estrato 3 , donde 17 eventos fueron en hombres $(6,75 \%)$ y 56 en mujeres (22,22\%); para un total de 73 casos $(28,98 \%)$. Este mis- 
estrato, sexo e intento mostró que en el estrato 2 los hombres intentan suicidarse en un porcentaje de 5,95\% (15 eventos) y las mujeres un 6,35\% (16 casos). Así mismo, en el estrato 3 se encontró que las mujeres que reportan intento de suicidio son 15 (5,95\%) y en hombres 9 casos (3,57\%), lo que coincide con otros reportes que cuales señalan que hay mayor vulnerabilidad en estratos socioeconómicos bajos $(6,33)$. Se abre un espacio para que otros estudios profundicen en el tema; debido a los factores que pueden afectar a esta población o a estratos más altos, donde los índices de suicidio consumado son mayores (6).

La prueba de Chi-cuadrado mostró relación estadísticamente significativa entre las variables de estudio: sexo, estrato bajo, situación descrita en estudios previos (17) y permanecer en el hogar, aspecto que se puede entender como exclusión social (18). La literatura evidencia que estas variables son factores que influyen en la presencia de la conducta suicida, como lo demuestra Ordoñez (11) en su investigación sobre suicidio consumado, la cual indica resultados similares en la relación de las variables sexo-estado civil, específicamente en Hombre-Estado civil, se evidencia que estas variables son factores que influyen en la presencia de la conducta suicida y hace un llamado a otras investigaciones para profundizar en el tema. Respecto al sexo, son las mujeres quienes muestran mayor proporción en la ideación y el intento con un $69,44 \%$ (175 casos) y los hombres un 30,56\% (77 casos), lo que coincide con hallazgos de investigaciones de la población en gene-

74 ral y para los adultos mayores donde el sexo femenino es el de mayor riesgo (22,
31). Otras publicaciones señalan que los intentos de suicidio se incrementan gradualmente en los grupos de mayor edad y alcanzan valores máximos en personas de 60 años o más $(15,30)$.

Existe evidencia del aumento en el tiempo del número de casos en la razón hombre: mujer en el suicidio consumado; lo que indica un aumento de la efectividad de los intentos en los hombres $(7,11$, $12,15)$, lo que sustenta los resultados obtenidos en la presente investigación, donde los adultos mayores presentan ideación e intento en las edades de 60 a 64 años en un mayor porcentaje, esto porque se evidencia más población en este grupo de población y un $\mathrm{OR}=1,098$ (IC 0,604 - 1,997) en adultos mayores de 63 años. Esto puede estar relacionado con que a medida en que aumentan de edad, los hombres van abandonando la ideación y el intento para ser más letales al consumar las acciones suicidas. Existe adicionalmente una limitante en estos datos y es que para el análisis se debe tener en consideración que los datos son porcentajes y no tasas. La ocupación con mayor presencia en los adultos mayores de esta investigación es el hogar con $54,77 \%$, lo que conlleva al cambio de rol del anciano, donde su productividad disminuye, la dependencia $y$ la sensación de soledad aumentan; en consecuencia, se deja de percibir al anciano como alguien que puede aportar económicamente y se desvirtúan sus capacidades físicas y mentales $(7,32,34)$, de esta manera y debido a los cambios en auto-concepto, desesperanza (15), su nuevo rol en la familia se convierte en un riesgo importante para el comportamiento suicida (32-34). 
En este análisis se encontró que el aseguramiento en salud de la población adulta mayor que presentó intento e ideación suicida en su mayoría pertenecen al régimen subsidiado con 49,60\% (125 casos), seguido del contributivo con $42,06 \%$ (106 casos) y vinculados con $4,37 \%$ (11 casos), este es un dato que no ha sido abordado en la literatura, lo que indica la necesidad de analizar el tema a través de otras investigaciones, las cifras distritales presentan mayores datos del régimen subsidiado respecto al contributivo, aspecto que se presenta como limitante para la base de datos analizada. Por otra parte, los hospitales que más reportan casos de ideación e intento suicida parece ser que es por las características sociodemográficas de la localidad. Frente a las variables sexo y estado civil en relación a la ideación e intento de suicidio, en este grupo se encontró que los hombres casados presentan el mayor porcentaje tanto para ideación con $6,75 \%$ (17 casos) como para intento con $4,76 \%$ (12 casos), cifras que no son similares a otros estudios en los que se señala que estar casado en los hombres se considera un factor protector $(15,31)$. Para las mujeres se presenta más ideación suicida, en las viudas con el $17,86 \%$ (45 casos) y los intentos en las casadas con $4,76 \%$ (12 casos), lo cual coincide con los resultados reportados en la literatura $(11,15)$. Es importante mencionar que Ordoñez (11) en su estudio sobre suicidio consumado reporta hallazgos similares a los encontrados en esta investigación, frente al estado civil en los hombres y las mujeres.

El suicidio es comprendido como un problema de salud pública, es entendido dentro de la categoría de lesiones como manifestación de violencia autoinflingida, siendo un reto para las naciones la disminución de sus cifras, esta situación es comprendida como evitable, su comprensión aportará a la disminución en la carga de la enfermedad y los costos que esta genera en los sistemas de salud (35). Desde la OMS se establecen lineamientos para la formulación de políticas públicas en salud para prevenir esta situación (36), este estudio servirá de insumo para la formulación de dichas políticas. Es un reto para futuras investigaciones el profundizar en las características de la población adulta mayor en relación a estrato socioeconómico, factores de riesgo y protectores, tanto en Bogotá cómo en otras ciudades de igual importancia en el país. Se señala la relevancia que tiene la base de datos Sisvecos de la Secretaría de Salud de Bogotá, ya que en ella se registran los casos presentados por año y son una fuente clave de información para futuras investigaciones, así mismo, una fuente de evidencia para el ajuste de políticas ya establecidas o para la creación de nuevas políticas que frente al tema requieren de evidencia para su soporte.

La información presentada en este trabajo comparte las características asociadas con el intento y la ideación suicida descrita en la literatura, con diferencias que pueden estar indicando nuevas fuentes de investigación para profundizar y adicionalmente para valorar, en el sentido de fortalecer la información recopilada para esta población. El presente estudio destaca como ventajas la identificación del riesgo por sexo, en particular en el adulto mayor, aspecto que reviste un problema de salud pública poco explo- 
rado en esta población; sin embargo, se identifica como limitantes el trabajar con fuentes de información secundaria, aspecto que genera problemas de calidad, en cuanto el registro y clasificación de la información $(37,38)$, el sistema de información Sisvecos se nutre del reporte de los hospitales públicos, aspecto que hace que se queden por fuera los estratos socioeconómicos más altos que muy seguramente acuden a consultas privadas y pertenecen al régimen contributivo. Además de no permitir la utilización de todas las variables de la base de datos y un tratamiento diferenciado a las variables ideación e intento suicida para realizar un análisis con otro tipo de pruebas estadísticas; adicionalmente, se reconoce el corto periodo de tiempo analizado, lo que llevó a una muestra no muy amplía.

\section{Conclusiones}

Los factores de riesgo sociodemográfico para intento e ideación suicida en este grupo de adultos mayores de 60 años de Bogotá, Distrito Capital, presenta significancia estadística en relación con estudio y sexo $\mathrm{P}=0,009$; estrato bajo $\mathrm{P}$ $=0,002$ y ocupación $\mathrm{P}=0,044$, presenta características similares a las reportadas en la literatura frente a sexo, ocupación y grupos etarios; caso contrario se evidencia en los resultados de las variables hombre-estado civil. Para esta población en particular, el riesgo sociodemográfico para ideación e intento suicida es 4,35 veces mayor en estratos socioeconómicos bajos respecto a los altos, las actividades fuera del hogar y vivir en pareja se identifican como factor protector.

\section{Agradecimientos}

Los autores agradecen a la Secretaría Distrital de Salud de Bogotá por el suministro de la información y a la Maestría en Salud Pública de la Fundación Universitaria del Área Andina por el apoyo brindado. Este es un trabajo que hace parte del proyecto internacional "Tentativa de suicidio en adulto mayor", adelantado desde el Centro Latino Americano de Estudos de Violência e Saúde Jorge Careli/ CLAVES da FIOCRUZ del Ministerio de Salud de Brasil, Rio de Janeiro, donde participan 14 ciudades de Brasil, Uruguay y desde de Colombia la Maestría en Salud Pública y Desarrollo Social de la Fundación Universitaria del Área Andina. 


\section{Referencias bibliográficas}

1. Nieto M, Alonso L. ¿Está preparado nuestro país para asumir los retos del planeta? Salud Uninorte. 2007;23(2):292-301.

2. Organización de las Naciones Unidas. Informe de la segunda asamblea mundial sobre el envejecimiento. [Internet]. Madrid; 2002 [citado 2015 dic. 06]. Disponible en: http://www.un.org/es/events/ pastevents/ageing_assembly2/

3. Reyes V, Romero M, Ramos M, CastiIlo A. Suicidio en el adulto mayor. Haban Cienc Méd. 2012;11(5):699-708.

4. Omran A. The epidemiologic transition; a theory of the Epidemiology of population change. Milbank men Fund Quart. 1971;49(4):509-38.

5. Departamento Administrativo Nacional de Estadística. Diagnóstico preliminar sobre personas mayor dependencia y servicios sociales en Colombia [Internet]. XXXX [citado 2015 dic. 06]. Disponible en: http:// www.minsalud.gov.co/proteccionsocial/.../ situacion $\% 20$ actual $\% 20$ de $\% 2$

6. World Health Organización. Preventing suicide: A global imperative. [Internet]. 2014 [citado 2014 sep. 09] http://apps.who.int/iris/bitstre am/10665/131056/1/9789241564779_ eng.pdf?ua $=1 \& u a=1$

7. Cendales R, Vanegas C, Fierro M, Córdoba R, Olarte A. Tendencias del suicidio en Colombia. Panam Salud Pública. 2007;22(4):231-38.

8. Organización Mundial de la Salud. Forenses [Internet]. 2004 [citado 2014 my. 22]. Disponible en: http://www.who.int/ mediacentre/news/releases/2004/pr61/es/

9. Rodríguez J, Medina O, Cardona D. Caracterización del suicidio en el departamento de Risaralda, Colombia. 2013;6(9):16.
10. Centro de Recursos para el Análisis de Conflictos, CERAC. Aproximación de la situación de violencia e inseguridad [Internet]. Bogotá: Katherine AA, Jorge R. 2005, jul. [citado 2014 my. 26]. Disponible en: http://www.cerac.org.co/pdf/LASUR-Baja.pdf

11. Ordoñez I. Caracterización de los suicidios en adultos mayores de 60 años ocurridos en Bogotá: 2003-2007 [Trabajo de grado Magister Scientiae en Salud Pública]. Bogotá: Universidad Nacional de Colombia; 2009.

12. Rodríguez JM, Rocha A, Mendieta G, Hidalgo A. Riesgo de muerte por suicidio en población colombiana 2000-2013. Ciênc. saúde coletiva [Internet]. 2018 nov. [citado 2019 febr. 28]; 23(11):398996. Disponible en: http://www.scielo. br/scielo.php?script=sci_arttext\&pi$d=S 1413-81232018001103989 \&$ Ing $=p t$

13. Instituto Nacional de Medicina Legal y Ciencias Forenses, Grupo Centro de Referencia Nacional sobre Violencia, SIRDEC. Sistema de información red de desaparecidos y cadáveres [Internet]. 2014. [citado 2015 ene. 16]. Disponible en: http://www.medicinalegal.gov.co/documents/88730/1656998/Forensis+Interactivo+2014.24-JULpdf.pdf/9085ad79d2a9-4c0d-a17b-f845ab96534b

14. Castillo J, García K, Pérez L, Cardona G. Caracterización de la conducta suicida en la localidad de Cuidad Bolívar en Bogotá [Trabajo de grado Psicología]. Bogotá: Universidad de La Sabana, Facultad de Psicología; 2012.

15. Velásquez J. Suicidio en el anciano. Rev colombiana de psiquiatría. 2013;43:80-4.

16. Doak M, Nixon A, Lupton D, Sthephen W. Self-poisoning in older adults: patterns of drug ingestion and clinical outcomes. Age and Ageing. 2009;38:407-11. 
17. Conwell Y, Orden K, Caine E. Suicidio en ancianos. Psiquiatría Biológica. 2012;19(4):127-36.

18. Ojagbemi A, Oladeji B, Abiona T, Gureje $O$. Suicidal behaviour in old age results from the Ibadan study of ageing. BMC Psychiatry.2013;13:80.

19. Traphagan JW. Interpretations of elder suicide, stress, and dependency among rural Japanese.Ethnology. 2004;43(4):315-29.

20. Fässberg $M$, et al. Functional disability and death wishes in older Europeans: results from the EURODEP concerted action. Soc Psychiatry Psychiatr Epidemiol. 2014;49(9):1475-82.

21. Ekramzadeh $S$, Javadpour A, Draper B, Mani A, Withall A, Sahraian A. Prevalence and correlates of suicidal thought and self-destructive behavior among an elderly hospital population in Iran. International Psychogeriatrics. 2012;24(9):1402-8.

22. Yan-Chiou K, Yun-Fang $T$, YanChiou L, Yea-Pyng L. Suicide experiences among institutionalized older veterans in Taiwan. The Gerontologist. 2009;49(6):746-54.

23. Peña D, Herazo M, Calvo J. Depresión en ancianos. Fac. Med. 2009;57(4):347-55.

24. Gonçalves CF, Minayo MC. Diferentes faces da depressao no suicidio em idosos. Ciencia\& Saúde colectiva. 2013;18(10):2985-94.

25. Ying-Jen Ch, Yun-Fang T, ShwuHua L, Hsiu-Lan L. Protective factors against suicide among young-old Chinese outpatients. BMC Public Health. 2014;14:372.

26. Bruce $M$, et al. Reducing suicidal ideation and depressive symptoms in depres-

78 sed older primary care patients. JAMA. 2004;291(9):1081-91.
27. Corte Constitucional de Colombia. Sentencia T-138/10 (febrero 24). Bogotá D. C.

28. Asociación Médica Mundial. Declaración de Helsinki [Internet]. XXXX [citado 2015 my. 20]. Disponible en: http://www. wma.net/es/30publications/10policies/b3/

29. Ministerio de Salud de Colombia. Resolución 008430 del 4 de octubre de 1993, por la cual se establecen las normas científicas, técnicas y administrativas para la investigación en salud.

30. Congreso Regional de Medicina Familiar Wonka Iberoamericana. X Seminario internacional de atención primaria de salud; Cuba: Facultad de Ciencias Médicas Miguel Enríquez; 2011.

31. Upegui H, Cabal G, Cortes C, Gallardo A, García M. Caracterización de la población con intento de suicidio en el Hospital Universitario del VaIle, Cali (1994-2010). Colomb Psiquiat. 2011;40(4):619-36.

32. Pérez S. Factores de riesgo suicida en el anciano. Ciencia \& Saúde colectiva. 2012;17(8):2011-16.

33. Núñez N, Olivera S, Lozada I. Perfil multidimensional de personas que han realizado intento de suicidio. Pensamiento psicológico. 2008;4(10):85-100.

34. Betacourt E. Caracterización psicosocial de ancianos que se suicidaron. Medisan. 2011;15(3):339-44.

35. Yang B, Lester D. Recalculating the economic cost of suicide. Death studies. 2007;31(4):351-61.

36. Organización Mundial de la Salud, Organización Panamericana de la Salud. Prevención del Suicidio: Un imperativo global. [Internet]. Washington DC. 2014. [citado 2017 my. 5]. Disponible en: http://apps.who.int/iris/bitstre am/10665/136083/1/9789275318508_ spa.pdf 
37. Comstock GW. Further comments on problems in death certification. Am J Epidemiol. 1986;124(2):180-1.
38. Celis A. El peatón lesionado en accidentes de tráfico de vehículo de motor: Mortalidad en México: 1985-1996. Gac Med Mex. 1999:135(3):353-58. 